\title{
Glutathione S-Transferase Alpha
}

National Cancer Institute

\section{Source}

National Cancer Institute. Glutathione S-Transferase Alpha. NCI Thesaurus. Code C107147.

A homo- or heterodimeric form of the glutathione S-transferase enzyme that is comprised of two alpha family subunits. 